\title{
Contribution of Transesophageal Echocardiography in the Etiological Diagnosis of Ischemic Stroke: Retrospective Study of 56 Cases
}

\author{
Ndiaye MB ${ }^{1}$, Mingou $\mathrm{JS}^{1 *}$, Sarr $\mathrm{SA}^{1}$, Aw F $\mathrm{F}^{1}$, Thiam $\mathrm{A}^{1}$, Bodian $\mathrm{M}^{1}$, Mbaye $\mathrm{A}^{2}$, Kane $\mathrm{Ad}^{3}$, Diao $\mathrm{M}^{1}$, Kane $\mathrm{A}^{4}$ and \\ $\mathrm{Ba} \mathrm{SA}^{1}$ \\ ${ }^{1}$ Department of Cardiology, Aristide Le Dantec Hospital University, Senegal \\ ${ }^{2}$ Department of Cardiology, Hospital General de Grand Yoff, Senegal \\ ${ }^{3}$ Department of Cardiology, Regional Hospital of Saint Louis, Senegal \\ ${ }^{4}$ Department of Cardiology, Dalal Jamm Hospital, Senegal
}

"Correspondence: Joseph Mingou, Department of Cardiology, Aristide Le Dantec Hospital University, Senegal

Received on 18 June 2019; Accepted on 03 September 2019; Published on 17 September 2019

Copyright (C) 2019 Ndiaye MB, et al. This is an open access article and is distributed under the Creative Commons Attribution License, which permits unrestricted use, distribution, and reproduction in any medium, provided the original work is properly cited.

\begin{abstract}
Introduction: Strokes constitute a public health problem. Ischemic strokes are caused by cardioembolic diseases in $20 \%$ of cases. We have realized this study in order to situate the role of transesophageal echocardiography in the search of cardioembolic diseases during ischemic strokes.

Materials and Methods: We have realized a retrospective study in the Cardiology Department of "Hopital Général de Grand Yoff" at Dakar, during two years from June 2003 to July 2005. Our patients received benefits from medical examination, biological exams, an ECG, a recording ECG, a transesophageal echocardiography, transthoracic echocardiography, a brain scan and echodoppler of neck vessels.

Results: Fifty-six cases have been identified. The average age was of $60.3 \pm 14.3$ years (extremes: 21-87 years). The sex-ratio (men over women) was of 0.75. The cardiovascular risk factors were dominated by blood hypertension and diabetes. On the standard electrocardiogram, we noted eleven cases of atrial fibrillation and one case of atrial flutter. Two patients have presented a paroxystic atrial fibrillation on recording electrocardiogram and six (10.7\%), either a significative stenosis or carotid thrombosis at neck vessels echodoppler. The board number $V$ is the summery of the etiology of ischemic strokes found by transesophageal echocardiography.
\end{abstract}

Keywords: strokes, transesophageal echocardiography, transthoracic echocardiography, thrombus, Dakar

\section{Introduction}

Stroke is a major public health problem because of its frequency, its mortality, the residual, physical and cognitive impairment that it causes, and the risk of ischemic, cerebral or myocardial recurrence to which it exposes [1-3]. 
Atherosclerosis and embolic heart diseases are responsible for the majority of ischemic strokes. Transesophageal echocardiography is now part of the diagnostic arsenal that is an essential complement to transthoracic cardiac ultrasound in the search for emboligenic heart disease.

The aim of this work was to situate the place of transesophageal echocardiography in the search for emboligenic heart disease in the event of ischemic stroke.

\section{Materials and Methods}

This is a retrospective study conducted in the Cardiology Department of Grand Yoff General Hospital in Dakar, over two years from June 2003 to June 2005.

All patients admitted for ischemic stroke were included in this work, based on an evocative clinical picture and a brain scan, during the study period, with no age limit.

Patients who did not receive transesophageal echocardiography were excluded from the study.

The studied parameters were age, gender, length of hospital stay, medical history (hypertension, diabetes, stroke, dilated cardiomyopathy, valvulopathy, complete arrhythmia by atrial fibrillation, ischemic heart disease).

Neurological signs: headache, lipothymia, Glasgow-Scale consciousness disorder (Table 1) [4], aphasia, dysarthria, motor deficit, tonic-clonic convulsions, facial palsy, were also studied.

\begin{tabular}{|l|l|l|}
\hline Eye response: $\mathbf{E}$ & Verbal response: $\mathbf{V}$ & Motor response: $\mathbf{M}$ \\
\hline spontaneously: 4 & oriented: 5 & obey commands: 6 \\
\hline to verbal command: 3 & confused: 4 & localizes pain \\
\hline to pain: 2 & inappropriate words: 3 & withdrawal from pain: 5 \\
\hline no eye opening: 1 & incomprehensible sounds: 2 & flexion to pain: 4 \\
\hline- & no verbal response: 1 & extension to pain: 2 \\
\hline- & - & no motor response: 1 \\
\hline
\end{tabular}

Table 1: Evaluation of consciousness disorders on the Glasgow Scale [4].

The cardiological signs such as dyspnea; classified according to the New York Heart Association, as represented (Table 2) [5], the shock of peak, the quivering, the arrhythmia, the breath, the noise of gallop, the rolling, were other parameters. All the others systems have also been examined.

\begin{tabular}{|l|l|}
\hline Classes & Signs \\
\hline I & No physical limitation in activity \\
\hline II & $\begin{array}{l}\text { Slight limitation in ordinary physical activity resulting in fatigue, } \\
\text { dyspnea, palpitations or angina }\end{array}$ \\
\hline III & $\begin{array}{l}\text { Marked limitation in activity, patients comfortable at rest but ordinary } \\
\text { activity leads to signs and symptoms }\end{array}$ \\
\hline IV & $\begin{array}{l}\text { Signs and symptoms present at rest, and any activity leads to increased } \\
\text { discomfort }\end{array}$ \\
\hline
\end{tabular}

Table 2: Classification of dyspnea according to New York Heart Association [5]. 
Laboratory assessment included a blood count, serum creatinine, prothrombin rate, international normalized ratio (INR), activated partial thromboplastin time, blood glucose, cholesterol (total cholesterol, HDL and LDL fractions) and triglycerides.

Our patients got benefited from a chest X-ray, a brain scan, an electrocardiogram, a long electrocardiographic recording, a Doppler ultrasound of the neck vessels and a transthoracic and multiplane transesophageal echocardiography probe.

Our data was entered on EPI INFO version 6.04 and analyzed using SPSS software version 9.05. We studied the sensitivity, specificity, positive and negative predictive values of transthoracic echocardiography compared to transesophageal echocardiography.

\section{Results}

Fifty-six ischemic stroke patients' cases were collected during the study period, i.e., $6.25 \%$ of hospitalizations (three transient ischemic attacks, fifty-three ischemic attacks, including hemorrhagic softening). The average age was 60.3 \pm 14.3 years (21-87 years) with a sex ratio (male to female) of 0.75 .

Cardiovascular risk factors were dominated by high blood pressure (thirty-six patients) and diabetes.

The admission examination noted thirty-nine cases of hemiplegia (69.6\%), six cases of facial palsy (10.7\%) and ten cases of arrhythmia.

Six patients (10.7\%) presented anemia at the laboratory assessment, and thrombocytopenia was noticed in two patients (3.5\%). Blood glucose was greater than or equal to $1.26 \mathrm{~g} / \mathrm{l}$ in eight patients $(20.5 \%)$. Total hypercholesterolemia was present in thirteen patients $(23.2 \%)$.

The LDL fraction was increased in seven patients (12.5\%) and the HDL fraction decreased in ten patients (17.8\%). Two cases $(3.5 \%)$ of hypertriglyceridemia were also found.

The electrocardiogram recorded eleven cases of complete arrhythmia by atrial fibrillation (19.6), including a paroxysmal and one case of atrial flutter.

Fifty patients (89.3\%) had results consistent with cerebral ischemic stroke, which also showed normal results in six patients $(10.7 \%)$. Six patients $(10.7 \%)$ had either significant stenosis or carotid thrombosis on the Doppler ultrasound of the neck vessels.

At $24 \mathrm{~h}$ electrocardiographic recording, two patients had paroxysmal atrial fibrillation.

Transthoracic echocardiography was normal in eighteen patients and non-contributory in eight cases (poor echogenicity).

The direct and indirect causes of ischemic strokes found respectively in transthoracic echocardiography and transesophageal echocardiography have been represented (Table 3 and 4) (Figure 1).

\begin{tabular}{|l|l|}
\hline Direct causes of systemic embolisms (number) & Indirect causes of systemic embolisms (number) \\
\hline Left atrial appendage thrombus 1 & Mitral stenosis 3 \\
\hline Left ventricle thrombus 1 & Dilated cardiomyopathy 4 \\
\hline & Spontaneous sludge 4 \\
\hline & Atrial septal aneurysm 1 \\
\hline
\end{tabular}

Table 3: Direct and indirect causes of systemic embolisms at transthoracic echocardiography. 


\begin{tabular}{|l|l|}
\hline Direct causes of systemic embolisms (number) & Indirect causes of systemic embolisms (number) \\
\hline Left atrial appendage thrombus 8 & Spontaneous sludge 19 \\
\hline Left ventricle thrombus 1 & Dilated cardiomyopathy 4 \\
\hline Athérome aortique protubérant 3 & Atrial septum aneurysm 3 \\
\hline & Mitral stenosis 3 \\
\hline
\end{tabular}

Table 4: Direct and indirect causes of systemic embolisms at transesophageal echocardiography.

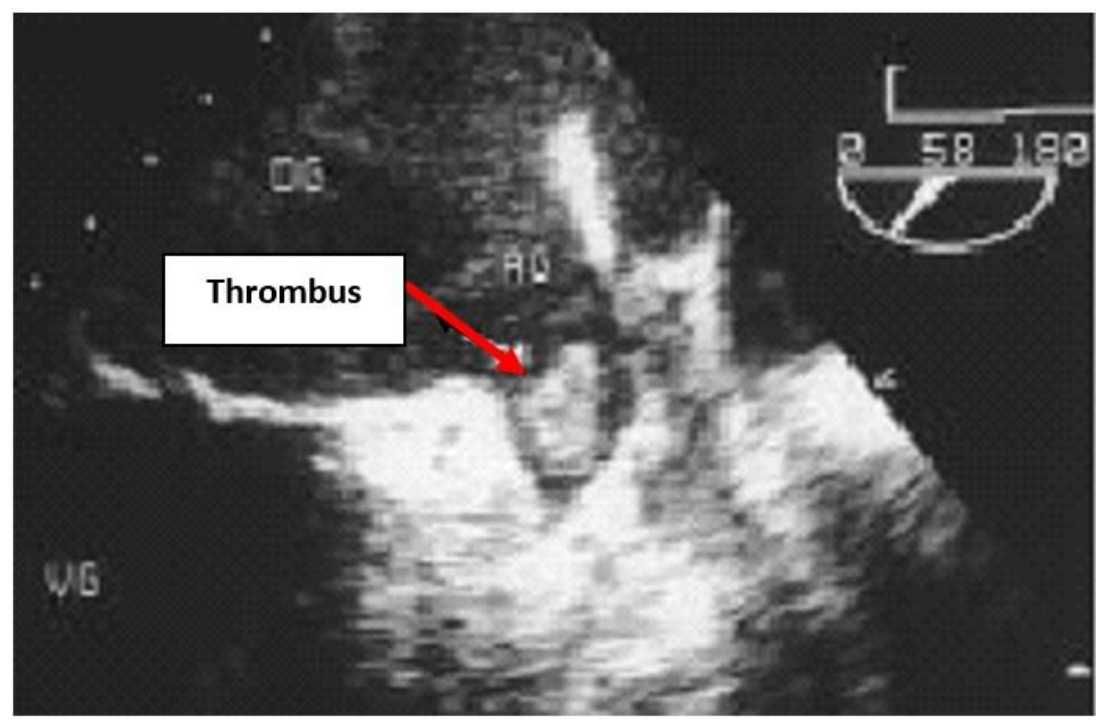

Figure 1: Transesophageal echocardiography, long axis 2 chambers, showing a thrombus (arrow) in the left atrial appendage. AU: left atrial appendage; OG: left atrium; VG: left ventricle.

Only $18.1 \%$ of direct causes of systemic embolism and $34.6 \%$ of indirect causes were found in transthoracic echocardiography compared to transesophageal echocardiography (Table 5).

\begin{tabular}{|l|l|l|}
\hline & TTE $(\%)$ & TOE $(\%)$ \\
\hline LAA spontaneous sludge & 3.5 & 30.3 \\
\hline LV spontaneous sludge & 3.5 & 7.1 \\
\hline Aortic spontaneous sludge & 0 & 17.8 \\
\hline LA thrombus & 1.8 & 14.3 \\
\hline LV thrombus & 1.8 & 1.8 \\
\hline Atrial septum aneurysm & 1.8 & 5.4 \\
\hline Protruding aortic atheroma & 0 & 5.4 \\
\hline
\end{tabular}

Table 5: Comparison of ischemic stroke causes found at transthoracic echocardiography (TTE) and transesophageal echocardiography (TOE). LAA: left atrial appendage; LA: left atrium; LV: left ventricle.

The sensitivity of transthoracic echocardiography in the search for embolic risk factors is $38 \%$, its specificity is $100 \%$, its negative predictive value is $100 \%$ and its positive predictive value is $65.2 \%$.

The sensitivity and specificity of transthoracic echocardiography according to potentially emboligenic pathologies has been represented (Table 6). 


\begin{tabular}{|l|l|l|}
\hline & TTE sensitivity (\%) & TTE specificity (\%) \\
\hline LAA spontaneous sludge & 11.7 & 100 \\
\hline LV spontaneous sludge & 50 & 100 \\
\hline LA thrombus & 12.5 & 100 \\
\hline LV thrombus & 100 & 100 \\
\hline Atrial septum aneurysm & 33.3 & 100 \\
\hline
\end{tabular}

Table 6: Sensitivity and specificity of transthoracic echocardiography (TTE) according to potentially emboligenic pathologies. LAA: left atrial appendage; LA: left atrium; LV: left ventricle.

\section{Comments}

The advanced age of stroke patients in our class (average age of $60.38 \pm 14.32$ years) is confirmed by Lannuzel et al. who reports an average population age of 69 years [6]. The female predominance found in our series, was not observed by Lannuzel et al. who noted a male predominance with a sex ratio of 1.2 [6]. Hypertension (64.3\% of patients) was the main risk factor in our series far exceeding the figures of several authors.

3-10\% of patients with ischemic stroke had a potential cardiac source of embolism detected by transthoracic echocardiography [7].

In our series, transthoracic echocardiography identified two direct causes and nine indirect causes of systemic embolism, while transesophageal echocardiography noted eleven direct and twenty-six indirect causes. In other words, only $18.1 \%$ of direct causes and $34.6 \%$ of indirect causes of systemic embolism were found in transthoracic echocardiography.

According to Touzé et al., approximately $20 \%$ of cerebral infarctions are the result of cardiac embolism [8].

In practice, apart from left intraventricular thrombosis and valvular vegetations, the cause of the embolic accident is rarely evidenced by transthoracic echocardiography.

Transesophageal echocardiography is superior to transthoracic echocardiography in detecting thrombi localized in the left atrium, with a sensitivity approaching $85 \%$, compared to 38-61\% for transthoracic echocardiography [9]. In our work, a thrombus located in the left atrium was detected in transthoracic echocardiography, compared to eight in transesophageal echocardiography. The interest of transesophageal echocardiography is major in the study of the left auricle, where the majority of thrombi is located [9]. In fact, a thrombus was noted in the left auricle 68 times in transesophageal echocardiography vs. once in transthoracic echocardiography [10]. Our results go in the same direction; indeed, no thrombus located in the left auricle found by transesophageal echocardiography had been detected by transthoracic echocardiography.

Spontaneous contrast is a marker of emboligenic risk and is usually visible in transesophageal echocardiography only. Thus, seventeen patients (30.4\%) had a spontaneous contrast in the left atrium in transesophageal echocardiography compared to two in transthoracic echocardiography in our series.

The three aneurysms of the inter-auricular septum discovered in transesophageal echocardiography in our series were not known by transthoracic echocardiography.

The role of ulcerated atheroma plaques and thromboses localized in the thoracic aorta in the occurrence of embolic accidents has been known for a long time [9]. Transthoracic echocardiography is of little interest here because of the difficulty in visualizing the walls of the thoracic aorta. On the other hand, transesophageal echocardiography allows excellent visualization of part of the ascending aorta, the aortic arch and the descending thoracic aorta. The risk of embolism is even greater when the lesions are pedicled, mobile, covered with thrombotic material [11-14].

Transesophageal echocardiography may also reveal spontaneous contrast in the thoracic aorta, which may also be an independent predictor of systemic embolic events. In a study, $28.6 \%$ of patients referred for embolic accident had a spontaneous contrast, against $13.8 \%$ of those examined for other reasons. 
The diagnostic efficiency of transesophageal echocardiography has been evaluated by several teams [7] (Table 7). It is constantly superior to transthoracic echocardiography, especially in the absence of clinical signs suggestive of cardiopathy.

The echocardiographic diagnosis remains reliable despite the development of radiological imaging techniques, electron beam scanning, helical CT or magnetic resonance imaging [7].

The diagnostic value of this technique has been specified [7]:

- The sensitivity: $100 \%$

- The specificity: $99 \%$

- The positive predictive value: $95 \%$

- The negative predictive value: $100 \%$

In our series, transthoracic echocardiography has a sensitivity of $38 \%$, a specificity of $100 \%$, a negative predictive value of $100 \%$ and a positive predictive value of $65.2 \%$.

Several studies have established the superiority of transesophageal echocardiography on transthoracic echocardiography for the detection of cardiac abnormalities that are directly or potentially emboligenic [15, 16].

\begin{tabular}{|l|l|l|l|}
\hline Author & Number of patients & LA thrombus TTE (\%) & LA thrombus TOE (\%) \\
\hline Aschenberg & 21 & 0 & 29 \\
\hline Daniel & 122 & - & 9 \\
\hline Krontzon & 19 & 0 & 26 \\
\hline Castello & 150 & 0 & 10 \\
\hline Black & 400 & 0 & 5 \\
\hline Lee & 50 & 0 & 10 \\
\hline Pearson & 79 & 0 & 6 \\
\hline Manning & 26 & - & 8 \\
\hline Olson & 21 & 0 & 10 \\
\hline Hwang & 213 & 7.5 & 14 \\
\hline Our series & 56 & 1.8 & 14.3 \\
\hline
\end{tabular}

Table 7: Left atrium thrombus detected by transthoracic echocardiography (TTE) and transesophageal echocardiography (TOE). LA: left atrium.

\section{Conclusion}

Transesophageal echocardiography is the most contributory examination in the etiological investigation of ischemic strokes in our series. It complements transthoracic echocardiography, which is very specific (100\%) but not very sensitive $(38 \%)$.

Our work confirms the key role of transesophageal ultrasound in the etiological investigation of ischemic stroke.

In view of these results we can recommend a cardiac ultrasound.

\section{References}

1. Bamford J, Sandercock P, Dennis M, et al. Classification and natural history of clinically identifiable subtypes of cerebral infarction. Lancet. 1991;337(8756):1521-26.

2. Hankey GJ, Warlow CP. Treatment and secondary prevention of stroke: evidence, costs, and effects on individuals and populations. Lancet. 1999;354(9188):1457-63. 
3. Murray CJ, Lopez AD. Mortality by cause for eight regions of the world: Global Burden of Disease Study. Lancet. 1997;349(9061):1269-76.

4. Danzizer N, Alamowitch S. Neurologie. 2nd ed. Paris: Med-Line ; 1997.

5. Marvin HM. Diseases of the Heart and Blood Vessels: Nomenclature and Criteria for Diagnosis. Arch Intern Med. 1964;113(6):906-07.

6. Lannuzel A, Salmon V, Mével G, et al. Épidémiologie des accidents vasculaires cérébraux en Guadeloupe et rôle du trait drépanocytaire. Rev Neurol. 1999;155(5):351-56.

7. Cohen A, Blanchard B, Chauvel C, et al. Complication of dobutamine stress echocardiography. Lancet. 1995;345(8943):201-02.

8. Touzé E, Varenne O, Calvet D, et al. Causes cardiaques d'embolie cérébrale. EMC - Neurologie. 2006;3(1):1-23.

9. Mirode A, Quéré JP, Tribouilloy C. Échographie transoesophagienne. Encycl Méd Chir, Elsevier, Paris, Cardiologie, 32-006-A-20, 2002, 20p.

10. Mugge A, Daniel WG, Angermann C, et al. Atrial septal aneurysm in adult patients. A multicenter study using transthoracic and transesophageal echocardiography. Circulation. 1995;91(11):2785-92.

11. Karalis DG, Chandrasekaran K, Victor MF, et al. Recognition and embolic potential of intra-aortic atherosclerotic debris. J Am Coll Cardiol. 1991;17(1):73-78.

12. Khatibzadeh M, Mitusch R, Stierle U, et al. Aortic atherosclerotic plaques as a source of systemic embolism. J Am Coll Cardiol. 1996;27(3):664-69.

13. Amarenco P, Cohen A, Hommel M, et al. Atherosclerotic disease of the aortic arch as a risk factor for recurrent ischemic stroke. N Engl J Med. 1996;334(19): 1216-21.

14. Tunick PA, Perez JL, Kronzon I. Protruding atheromas in the thoracic aorta and systemic embolization. Ann Intern Med. 1991;115(6):423-27.

15. Black IW, Hopkins AP, Lee LC, et al. Role of transoesophageal echocardiography in evaluation of cardiogenic embolism. Br Heart J. 1991;66(4):302-07.

16. Mirode A, Tribouilloy C, Adam MC, et al. Contribution of transesophageal ultrasonography in the etiologic evaluation of a systemic embolic accident. Apropos of 451 patients. Arch Mal Coeur Vaiss. 1993;86(12):1701-08. 